\title{
Task-Related Interaction between Basal Ganglia and Cortical Dopamine Release
}

\author{
Gaëtan Garraux, ${ }^{1,3,4}$ Philippe Peigneux, ${ }^{3}$ Richard E. Carson, ${ }^{2}$ and Mark Hallett ${ }^{1}$ \\ ${ }^{1}$ Human Motor Control Section, National Institute of Neurological Disorders and Stroke and 2 Positron Emission Tomography Department, Clinical Center, \\ National Institutes of Health, Bethesda, Maryland 20892, and ${ }^{3}$ Cyclotron Research Center and ${ }^{4}$ Department of Neurology, University of Liège, B-4000 Liège, \\ Belgium
}

Dopamine (DA) is a powerful neuromodulator for a wide variety of behaviors. Considerable evidence accumulated from rodent and monkey experiments over the last two decades suggests that DA activity in the frontal cortex is reciprocally linked to that in functionally related basal ganglia (BG) structures. However, the functional importance of this in humans is still unknown. To address this issue, we measured endogenous DA release using positron emission tomography in 15 healthy subjects as they practiced the first training session of a finger sequence learning task. Significant results were observed not only in striatal areas but also in extrastriatal "motor" regions, bilaterally. Faster learning was specifically coupled to lower DA release in the sensorimotor part of the globus pallidus pars interna (GPi) contralateral to the moving hand, which was paralleled by a higher increase in DA levels in the pre-supplementary motor area (pre-SMA). This finding provides original evidence supporting a motor-learning-related interaction between DA release in left GPi and pre-SMA, a mechanism that may also apply to other anatomically and functionally interconnected BG and frontal cortical areas as a function of behavior.

Key words: dopamine; PET; ${ }^{11} \mathrm{C}$-raclopride; pallidum; SMA; motor skill learning

\section{Introduction}

Dopamine (DA), one of the major slow-acting brain neurotransmitters in the mammalian brain, is a powerful modulator for many behaviors. Dysfunctions of DA systems underlie aspects of various neuropsychiatric and movement disorders such as schizophrenia and Parkinson's disease. A better knowledge of the mechanisms by which behavior is modulated by DA in humans is a prerequisite for improving treatment outcomes of these disorders.

In primates, mesencephalic DA neurons project to both the basal ganglia (BG) (Jan et al., 2000; Joel and Weiner, 2000) and frontal cortex (Williams and Goldman-Rakic, 1998). Considerable evidence accumulated from pharmacological and behavioral experiments over the last two decades suggests that DA release in the former is reciprocally linked to that in the

\footnotetext{
Received April 10, 2007; revised 0ct. 23, 2007; accepted 0ct. 25, 2007.

This work was supported in part by the Intramural Research Program of the National Institute of Neurological Disorders and Stroke (NINDS)-National Institutes of Health (NIH). G.G. was also supported by grants from the Fondation Horlait-Dapsens, the Belgian American Educational Foundation, NATO, NINDS, and the Fondation Léon Frédéricq (University of Liège). P.P. was supported by PAI/IAP Interuniversity Pole of Attraction P5/04. G.G. is a research associate at the Fonds National de la Recherche Scientifique Belge. We thank Nguyet Dang for technical assistance; PET Technicians of the Clinical Center; NIH, for their expert help with scanning; and Devera Schoenberg for skillful manuscript editing.

Correspondence should be addressed to Dr. Mark Hallett, Human Motor Control Section, National Institute of Neurological Disorders and Stroke, National Institutes of Health, Building 10, Room 5N226, 10 Center Drive, MSC 1428, Bethesda, MD 20892-1428. E-mail: hallettm@ninds.nih.gov.

P. Peigneux's present address: Department of Neurospychology, Free University of Brussels (ULB), Brussels, Belgium.

R. E. Carson's present address: Yale Positron Emission Tomography Center, New Haven, СT 06520.

D0I:10.1523/JNEUROSCI.1595-07.2007

Copyright $\odot 2007$ Society for Neuroscience $\quad 0270-6474 / 07 / 2714434-08 \$ 15.00 / 0$
}

latter (Pycock et al., 1980; Duvauchelle et al., 1992; Roberts et al., 1994; Kolachana et al., 1995; Iwano et al., 1997; Wilkinson et al., 1998). In humans, a regionally specific, long-lasting increase in BG DA release was reported after nonphysiologic frontal cortex magnetic stimulation (Strafella et al., 2001, 2003), although a concomitant change in DA levels in the stimulated area was not assessed.

One critical aspect of behavior that is modulated by DA is motor learning (Matsumoto et al., 1999; Reynolds et al., 2001). Therefore, we measured motor-learning-related changes in extracellular DA levels simultaneously in BG and caudal frontal cortical areas compared with a baseline task and investigated whether DA release in interconnected BG and cortical areas shows an opposite relationship with behavior. The difference in DA levels between tasks was estimated by measuring inverse variations in ${ }^{11} \mathrm{C}$-raclopride (RAC) binding potential (BP) to $\mathrm{D}_{2} / \mathrm{D}_{3}$ dopaminergic receptors using positron emission tomography (PET) (Koepp et al., 1998; Laruelle, 2000).

From a behavioral point of view, learning is difficult to assess per se. A change in knowledge (i.e., behavioral performance) at the end of training is usually taken as evidence for learning. Instead, we assessed motor skill learning by measuring the rate of performance improvement in the course of training, a feature that may be critically sensitive to DA (Reynolds et al., 2001). More precisely, motor learning speed was estimated by the rate of reaction time (RT) improvement during the serial reaction time task (SRTT) (Fig. 1a) (Nissen and Bullemer, 1987). Unbeknownst to the participants, the sequence (SEQ) of successive visual stimuli was governed by the probabilistic rules of an arti- 
a)

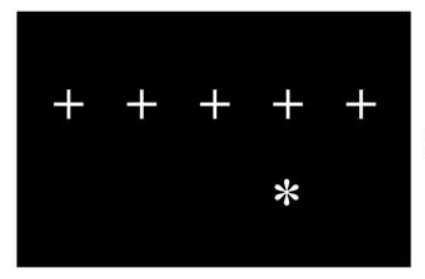

b)

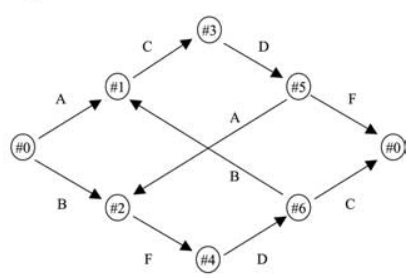

c)

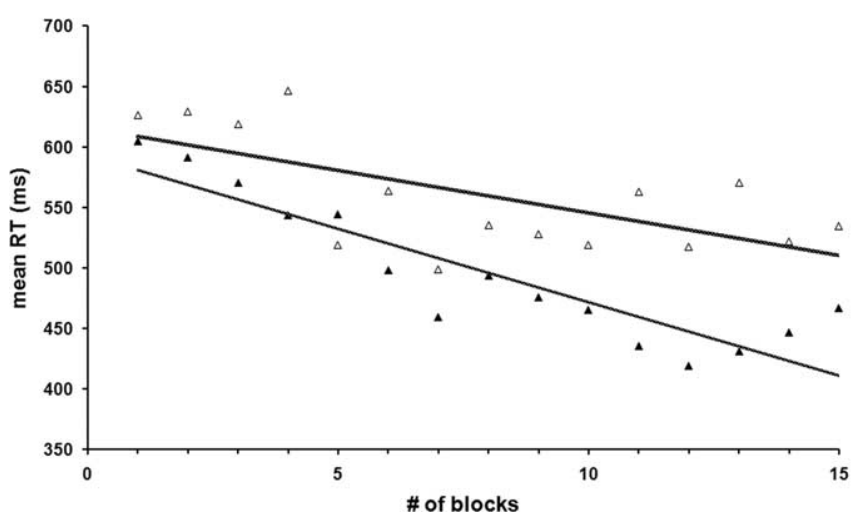

Figure 1. Motor learning task. $\boldsymbol{a}$, The SRTT was designed as a five-alternative spatial choice reaction time task involving individual finger motor responses with the dominant hand. Subjects were instructed to respond to the appearance of a visual stimulus (star) below the marker (cross) by pressing the spatially congruent key with the corresponding finger as fast and as accurately as possible. One practice block equals 205 trials. $\boldsymbol{b}$, Artificial grammar used to control the sequence of successive stimuli in the probabilistic serial reaction time task [adapted from Jiménez et al. (1996)]. Each label (A, B, C, D, and E) corresponds to a stimulus location on the computer screen. At each node (\#), one of the arrows is randomly selected. In this setting, the appearance of any SEQ stimulus not only defines the expected finger move but also provides the subject with contextual information that could be used in advance to prepare the next response by weighing its probability of (non)occurrence (Lungu et al., 2004). Hence, the gradual improvement in response time over practice depends on improved rule-based selection of the most likely forthcoming motor responses before the appearance of the upcoming stimulus (i.e., higher motor response anticipation). To assess learning-specific response time improvement, $15 \%$ of SEQ stimuli within a block of practice (205 trials) were randomly replaced by RAND stimuli, which violated the rules of the grammar. c, Behavioral data collected during PET in a representative subject. Block averaged RT for RAND (empty triangles) and SEQ (filled triangles) stimuli are expressed as a function of the number of blocks performed during the RAC PET scan (Fig. 2). The lines represent the linear regressions fitting the gradual decrease in RT for RAND (top trace) and SEQ (bottom trace) stimuli. The slope for SEQ stimuli (linear change in RT, -12.2 ms per block) was steeper than that for RAND stimuli (linear change in RT, $-7.1 \mathrm{~ms}$ per block). Motor sequence learning speed (DIFF_SLOPE) was estimated by computing the difference in the slopes between RAND and SEQ stimuli (for this individual, DIFF_SLOPE was equal to -7.1 $+12.2=5.1 \mathrm{~ms}$ per block). Following this convention, a higher, positive value for DIFF_SLOPE indicated faster finger sequence learning.

ficial grammar (Fig. 1b) (Jiménez et al., 1996). By randomly embedding stimuli that violated the grammatical rules [random (RAND) stimuli] with SEQ stimuli, we simultaneously monitored the rate of changes in RT for SEQ compared with RAND stimuli across practice blocks. Any RT improvement for RAND stimuli presumably involved nonspecific processes, including visuomotor adaptation within the limit of preexisting motor gain mechanisms, or general learning effects unrelated to the specifics of the sequential material. Hence, motor sequence learning speed was computed as the difference in the rate of RT improvement between RAND and SEQ stimuli (Fig. 1c). This difference (labeled as DIFF_SLOPE) was used as the dependent variable in the correlation analysis with PET data.

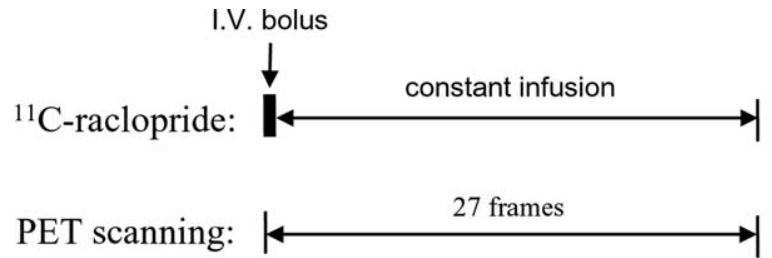

Behavior:

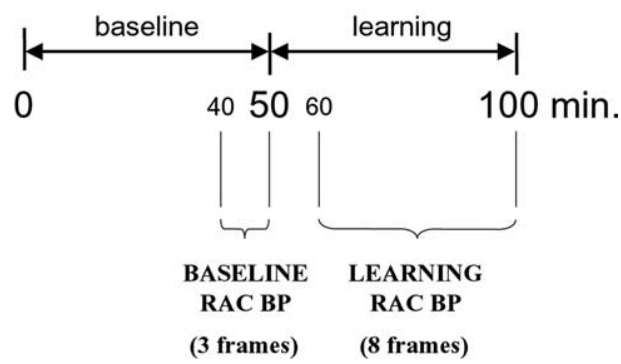

$\begin{array}{cc}\text { BASELINE } & \text { LEARNING } \\ \text { RAC BP } & \text { RAC BP } \\ \text { (3 frames) } & \text { (8 frames) }\end{array}$
Figure 2. PET scanning timeline. ${ }^{11} \mathrm{C}$-raclopride was administered for 100 min using a bolusplus-infusion protocol. PET scanning began at the start of tracer infusion and proceeded for 100 min. Parametric images of RAC BP during BASELINE and LEARNING conditions were created using frames $14-16$ and $20-27$, respectively (see Materials and Methods).

\section{Materials and Methods}

\section{Study design}

Participants were told that the main goal of the experiment was to study the cerebral effects of sustained practice on a simple choice reaction time task over 2 consecutive days. On the first day, cerebral RAC BP was measured in 15 healthy subjects (seven females; mean age, 59.2; range, 47-70 years) using a single 100 min PET scan under two consecutive experimental conditions of 50 min duration each (Fig. 2): an oculomotor sequence task (BASELINE) and the SRTT (LEARNING). A pilot behavioral study conducted in six healthy subjects (two females, four males; mean age, 55.3; range, $46-63$ years) had previously indicated that significant task learning could be achieved within the time window imposed by PET. The age range of our subjects was originally chosen in expectation of another study involving a comparison with a patient group.

RAC BP was measured during the early learning stage, which is defined by a large performance improvement within the first training session compared with changes occurring at a slower rate later on (Karni et al., 1998). This was verified here by comparing behavioral performance in the PET scanner with that measured during a second training session on the following day outside the PET scanner. Immediately after completing the last learning block on the second learning day, subjects were debriefed about the SRTT, and then they practiced a GENERATION task to assess the level of explicit knowledge about the material they had learned.

\section{Subjects}

All but one of the participants were right-handed according to the Edinburgh Inventory (Oldfield, 1970). None had any history of neurological disorder, head trauma with loss of consciousness, epilepsy, brain surgery, systemic illness, or excessive drug or alcohol consumption. Informed verbal and written consent for this research protocol, which was approved by the National Institute of Neurological Disorders and Stroke Institutional Review Board and the National Institutes of Health Radiation Safety Committee, was obtained from all subjects. Participants were instructed not to drink any beverages containing caffeine or alcohol within $24 \mathrm{~h}$ before the experiment.

\section{Task procedure}

In all conditions, participants faced a computer screen, positioned $\sim 1 \mathrm{~m}$ from their eyes, on which five permanent position white markers were displayed horizontally on a black background throughout the experiment. The dominant hand rested on an ergonomic five-button response unit (MRI Devices Corporation, Waukesha, WI). There was a one-toone mapping between a marker and a response key.

In the BASELINE condition, subjects were asked to pay attention to 
the appearance of a visual stimulus (white star) sequentially displayed for $300 \mathrm{~ms} 2 \mathrm{~cm}$ below one of the markers according to a pseudorandom sequence. No hand movement was required. The interstimulus interval (ISI) was kept constant at $700 \mathrm{~ms}$ so that the rate of presentation of the successive stimuli was $1 \mathrm{~Hz}$. After 205 trials, subjects rested for $30 \mathrm{~s}$. The BASELINE condition started $\sim 3$ min before tracer injection and continued until 50 min after scanning onset.

In the LEARNING condition, the apparatus was the same as in the BASELINE condition, but subjects were instructed to respond to the appearance of the visual stimulus by pressing the spatially congruent key with the corresponding finger as quickly and as accurately as possible. The stimulus was displayed until the subject pressed a response key, thereby minimizing working memory requirements. The interval between the subject response and the display of the following stimulus [response-to-stimulus interval (RSI)] was held constant at $250 \mathrm{~ms}$ throughout the experiment (Peigneux et al., 2000). If subjects pressed the wrong key, the computer program registered an error, and a brief error message was displayed on the screen. A single practice block consisted of 205 successive trials. After completing each block, subjects rested for $30 \mathrm{~s}$, during which the mean global reaction time and accuracy level during the last performed block were displayed. Subjects were then encouraged to improve their overall RT in the next block while keeping overall accuracy at $95 \%$. Because the overall visuomotor response speed is typically variable across participants, and the RSI was held constant in the present task (see above), the level of practice (i.e., number of blocks performed) within the fixed 50 min PET scanning was expected to differ between subjects.

Unbeknownst to the participants, the sequential structure of visual stimuli in the LEARNING condition was manipulated by the experimenter. In brief, the SEQ of successive stimuli was controlled by a finite state grammar adapted from Jiménez et al. (1996). To assess whether participants were sensitive to the sequential structure of the material, $15 \%$ of SEQ stimuli were randomly replaced by RAND stimuli that violated the rules of the artificial grammar. Additional details on the procedure were published previously (Peigneux et al., 2000). Before the PET scan, all subjects were trained using a set of 60 unrecorded trials in which the series of successive stimuli followed a pseudorandom sequence.

On the day after PET, 14 of 15 subjects further practiced the SRTT outside the scanner for $\sim 60 \mathrm{~min}$. After completing the last learning block, subjects were then examined to assess the level of explicit knowledge about the material they had learned. They were first asked whether they had noticed anything about the sequence of stimuli. Thereafter, they were told that a set of complicated rules had been used to determine the sequence locations at which the successive stimuli occurred. Participants were then exposed to a GENERATION task in which they faced the same apparatus as that used for the SRTT, but in this case, they were instructed to predict, without time constraint, the location of the next stimulus by pressing on the corresponding key (Peigneux et al., 2000). A total of 205 prediction trials were recorded.

Visual stimuli were generated, and subjects' responses were recorded by a personal computer using COGENT cognitive interface software (COGENT 2000; Wellcome Department of Imaging Neuroscience, London, UK) implemented in Matlab 5.3 (MathWorks, Sherborn, MA).

\section{Behavioral data analysis}

Behavioral data were analyzed off-line using Matlab. In each trial, the response time was defined as the interval between the display of a stimulus on the computer screen and the hit of the response key. For each block (205 trials) and subject, the mean accuracy and mean RT in the context set by the previous stimulus were computed in parallel for RAND and SEQ stimuli. Incorrect responses and extreme values falling outside two SDs from the block mean were discarded from the RT data analysis. The mean group rejection rate over blocks $( \pm S D)$ was $5.6 \pm 0.9 \%$.

To accommodate for within-subject RT variability for SEQ and RAND stimuli across blocks (Peigneux et al., 2000) and methodological constraints inherent to RAC PET, the individual rate of changes in RT for SEQ and RAND stimuli was fitted using linear regressions. Finger sequence learning speed was estimated as the difference in the linear slope coefficients modeling the rate of change in RT across blocks between RAND and SEQ stimuli and labeled DIFF_SLOPE.

In the generation task, the outcome measure was the prediction accuracy given the context set by the previous stimulus. The group mean prediction accuracy was compared with that from 15 computerized simulations of continuous random prediction (Peigneux et al., 2000).

\section{Imaging data acquisition}

Subjects underwent a single dynamic PET acquisition using a GE advance PET tomograph (General Electric Medical Systems, Milwaukee, WI) in the 3D scanning mode (septa retracted) with a total axial field of view of $14.8 \mathrm{~cm}$, producing a reconstructed resolution of $6-7 \mathrm{~mm}$ in all directions.

RAC was used as a tracer to examine task-related changes in DA (Laruelle, 2000). The injection time was between 11:00 A.M. and 2:00 P.M. for all subjects; scanning began at the start of tracer infusion, generating 27 time frames of $30 \mathrm{~s}$ to $5 \mathrm{~min}$ epochs over $100 \mathrm{~min}$ (Fig. 2). Head movements were minimized by using a thermoplastic mask molded around the subject's head. An 8 min transmission scan was obtained before tracer injection for attenuation correction.

RAC was delivered in a $20 \mathrm{ml}$ syringe and administered as a $1 \mathrm{~min}$ bolus followed by a constant infusion [bolus-plus-infusion (B/I)] using a computer-operated pump (Harvard Instruments, Natick, MA). In the $\mathrm{B} / \mathrm{I}$ paradigm for RAC administration, the ligand-receptor equilibrium can be measured in the brain before and after an experimental manipulation in a single scan (Carson, 2000). In the current experiment, the bolus volume was $51 \%$ of the total volume injection in the syringe, as previously recommended (Watabe et al., 2000). Because of radioactivity decay, the bolus accounted for $79 \%$ of the radiation dose. Accounting for the decay, the mean activity effectively injected was $17.1 \mathrm{mCi}$ (range, $10.6-20.8 \mathrm{mCi})$.

In addition to PET scanning, 13 of 15 participants underwent a highresolution volumetric anatomical magnetic resonance imaging (MRI) of the brain on a 3.0 T GE Signa system (General Electric Medical Systems) using a T1-weighted inversion recovery fast-spoiled gradient-echo sequence (repetition time, $8.2 \mathrm{~ms}$; echo time, $3.3 \mathrm{~ms}$; inversion time, 725 $\mathrm{ms}$; flip angle, $6^{\circ}$; matrix size, $256 \times 256$; yielding 124 contiguous axial slices with a thickness of $1.3 \mathrm{~mm}$ and in-plane resolution of $0.97 \times 0.97$ $\mathrm{mm})$.

\section{Imaging data preprocessing}

Corrections for subject motion during the $100 \mathrm{~min}$ PET acquisition were performed with a mutual information registration of each time frame to a standard frame (10-15 min after injection) before attenuation correction. Based on the calculated motion, the transmission images were resliced and projected for final attenuation correction, reconstruction, and realignment.

Subsequent off-line PET data preprocessing was performed using the Statistical Parametric Mapping Software (SPM2; Wellcome Department of Imaging Neuroscience; freely available at http://www.fil.ion.ucl.ac.uk/ spm) implemented within Matlab 6.1 (MathWorks). Image volumes were initially checked for gross artifacts and manually reoriented to grossly conform to the orientation of the interhemispheric fissure and intercommissural plane of the canonical template image proposed as default in SPM2. The center point was approximately placed on the anterior commissure. Because visual inspection of data suggested residual frame misalignment despite the initial realignment procedure (see above), individual time series were subsequently realigned to the corresponding 10-15 min postinjection frame using a mutual normalized information algorithm implemented in SPM2. This SPM2 algorithm was also used to anatomically coregister individual skull stripped MRI (created using mricro, freely available at http://www.psychology.nottingham.ac.uk/staff/crl/mricro.html) images with their respective image time series using images of total summed tracer activity.

\section{Imaging data analysis}

The BP was determined from concentration ratios in regions with and without specific binding (Carson, 2000). Under equilibrium, the specific-to-nonspecific equilibrium partition can be obtained directly from the radioactivity concentration of the receptor-target regions (C) 
and a receptor background region $\left(\mathrm{C}^{\prime}\right)$ as the ratio $\mathrm{BP}=\mathrm{V}_{3}{ }^{\prime \prime}=(\mathrm{C}-$ $\mathrm{C}^{\prime}$ )/C. BP equals the equilibrium ratio of bound ligand to free plus nonspecifically bound tracer under the assumptions that nonspecific binding is uniform throughout the brain.

In the present experiment, $C^{\prime}$ was calculated from volume-of-interest (VOI) values averaged over a few scan frames in the cerebellum using a commercially available software (PMOD; PMOD Technologies, Zürich, Switzerland), whereas $C$ was computed by pixel-by-pixel averaging over several whole-brain image frames. Cerebellar activity was measured from two elliptic VOIs $\left(6.7 \mathrm{~cm}^{3}\right.$ each) placed over the cerebellar hemispheres. After the optimization procedure proposed by Watabe et al. (2000), C and $\mathrm{C}^{\prime}$ in the BASELINE and LEARNING conditions were calculated by averaging over three frames over 40-50 min and over eight frames over 60-100 min, respectively (Fig. 2). Thus, two condition-specific parametric images of RAC BP were created for each subject. These 30 images were spatially normalized using a custom-made symmetrical RAC template in Montreal Neurological Institute space (http://www.bic.mni.mcgill.ca) as recommended previously (Meyer et al., 1999). Each spatially normalized, condition-specific, parametric RAC BP image was resliced to a final voxel size of $3 \times 3 \times 3 \mathrm{~mm}$ and smoothed using an $8 \mathrm{~mm}$ full-width at half-maximum Gaussian kernel. Data from the left-handed subject who performed the motor learning task with the left hand were right-left flipped so that the hemisphere contralateral to the moving hand was on the left in all subjects.

Voxel-by-voxel analysis. Processed RAC BP images were analyzed voxelwise using SPM2 in the framework of the general linear model.

A paired $t$ test looking for a decreased RAC BP during LEARNING compared with BASELINE (i.e., DA release) was initially performed. No global BP normalization procedure was performed. Results were characterized in terms of the probability that the difference in magnitude value in intensity of a given voxel could occur by chance under the null hypothesis.

For the voxel-by-voxel correlation analysis between brain imaging and behavioral data across subjects using SPM2, a single image representing the magnitude of the difference in RAC BP between conditions (BASELINE minus LEARNING) was first created for each participant. The 15 resulting images were then entered into a single SPM2 design matrix including DIFF_SLOPE and a constant term as the regressors. After model estimation, brain areas in which RAC displacement showed a positive association with DIFF_SLOPE were determined by applying a $\mathrm{t}$-contrast that was +1 (i.e., positive correlation between DA release and learning speed). Brain areas showing a negative association between RAC displacement and DIFF_SLOPE (i.e., negative correlation between DA release and learning speed) were searched for using a t-contrast that was -1 . Results were characterized in terms of the probability that the correlation between behavioral performance and the value in a given voxel could occur by chance under the null hypothesis.

Significance level was set at $p<0.05$ after familywise error (FWE) correction for multiple comparisons in the entire volume of analysis. The correction was limited to a 5-mm-radius spherical volume [small volume correction (SVC)] around regions in which an effect was hypothesized, namely BG and cortical motor-learning-related areas (i.e., caudal frontal cortex). This approach is commonly accepted in the neuroimaging community (Thieben et al., 2002). Anatomical localization was performed by superimposing the maxima foci identified by the $\operatorname{SPM}\{\mathrm{t}\}$ on the mean spatially normalized MRI image (avMRI) from all study subjects for whom an anatomical MRI was available $(n=13)$ and by reference to proposed landmarks when appropriate (Picard and Strick, 1996). This approach has obvious limitations, and the designation of the anatomical structures should be viewed as tentative rather than definitive.

Post hoc VOI approach. In regions identified by the SPM analysis, individual BP values were extracted from the 30 spatially normalized unsmoothed parametric BP images using a unique set of VOIs created within PMOD. Striatal VOIs were manually drawn on the avMRI using the same procedure as that described by Mawlawi et al. (2001). Postcommissural globus pallidus pars interna (GPi) and premotor VOIs were derived from the Wake Forest University PickAtlas (WFU_PickAtlas freely available at http://www.fmri.wfubmc.edu/download.htm) and manually edited using PMOD to fit the corresponding structure as de-
Table 1. Comparison of learning performance between days 1 (PET) and 2

\begin{tabular}{llll}
\hline & Slope RAND (SD) & Slope SEQ (SD) & $\begin{array}{l}\text { Motor sequence } \\
\text { learning speed }\end{array}$ \\
\hline Day 1 & $-11.84^{*}(20)$ & $-15.77^{*}(19.6)$ & $3.93^{* *}$ \\
Day 2 & $-1.23(3.9)$ & $-2.81^{*}(3.6)$ & $1.58^{* * *}$ \\
\hline
\end{tabular}

The data represent group mean values $(n=14) .{ }^{*}$ Slope significantly lower than zero $(p<0.05) .{ }^{* *} p=0.0001$ paired $t$ test; ${ }^{* * *} p=0.01$, paired $t$ test.

${ }^{a}$ Difference in RT improvement between RAND compared with SEQ stimuli expressed in milliseconds per practice block (see Materials and Methods).

fined on the avMRI. The mean VOI size across regions was $1485 \mathrm{~cm}^{3}$ (range, 597-4063).

\section{Results \\ Behavior}

Pilot experiment

Results from the pilot behavioral experiment in six subjects demonstrated that a significant improvement in RT could be achieved within the first training session as shown by a significantly negative value for both mean group linear slope coefficients (RAND stimuli: $-6.95 \pm 5.24, p=0.02$; SEQ stimuli: $-9.94 \pm 4.9, p=$ $0.004)$. In other words, using a linear model, the rate of improvement in RT in this group was $\sim 3$ ms higher per practice block for SEQ compared with RAND stimuli. This corresponds to an average gain in the rate of RT improvement of $\sim 43 \%$ for SEQ compared with RAND stimuli.

\section{RAC PET study}

Fifteen individuals naive to the SRTT were enrolled in the PET experiment. As expected (see above), the number of blocks performed within the fixed 50 min PET scanning differed between subjects (group mean $\pm \mathrm{SD}=13.3 \pm 2$ blocks). The individual accuracy averaged over blocks was $97 \%$ for both stimuli types in all subjects.

RT for RAND and SEQ stimuli both improved with practice. Nevertheless, the group mean slope fitting the gradual decrease in RT across blocks was significantly steeper for SEQ compared with RAND stimuli (mean slope coefficient, $-15.0 \pm 19.1 \mathrm{vs}-11.5 \pm$ 19.3). This difference was significant (paired $t$ test, $p=0.0005$ ) and in the same range as that observed in the pilot experiment (gain, 30.5\%). Additional analyses indicated that DIFF_SLOPE was not related with age in our sample (Pearson's $r=0.16, p=$ $0.58)$, nor was it proportional to the level of practice within the fixed PET scanning time (i.e., number of blocks performed during the PET scan; Pearson's $r=-0.18 ; p=0.54$ ), which results from inevitable variations in overall visuomotor response speed across subjects (see above). This last result suggests that DIFF_SLOPE, the behavioral measure we have used for correlation with imaging data, is not dependent on the number of finger movements during PET scanning.

\section{Follow-up}

On the following day, 14 subjects further practiced on average 16 blocks of the motor sequence learning task outside the PET scanner. Although the group mean RT for SEQ still gradually improved on day 2, the rate of improvement was lower than on day 1 (Table 1). An ANOVA of learning slopes showed a significant effect of the day of testing (steeper slopes on day 1 than on day 2; $F=5.25 ; p=0.03$ ) and stimuli type (steeper slopes for SEQ than RAND stimuli; $F=30 ; p=0.0001)$ as well as an interaction between these factors $(F=8.19 ; p=0.01)$. This result suggests that RAC PET measurements were obtained in a temporal window dominated by the early stage of motor learning characterized 
by a relatively large improvement in performance (Karni et al., 1998).

Afterward, subjects were made aware that a set of complicated rules was used to control the sequence of stimuli. They were then administered a generation task in which they had to predict the location of the next stimulus by pressing on the corresponding key (Peigneux et al., 2000). Generation performance was at chance level $(p=0.19)$, indicating that participants did not gain valuable explicit knowledge about the sequential structure of the material learned during task practice.

\section{Analysis of parametric RAC BP images Learning-related decrease in RAC BP \\ Parametric RAC BP images representing} BASELINE and LEARNING conditions were compared using a voxelwise paired $t$

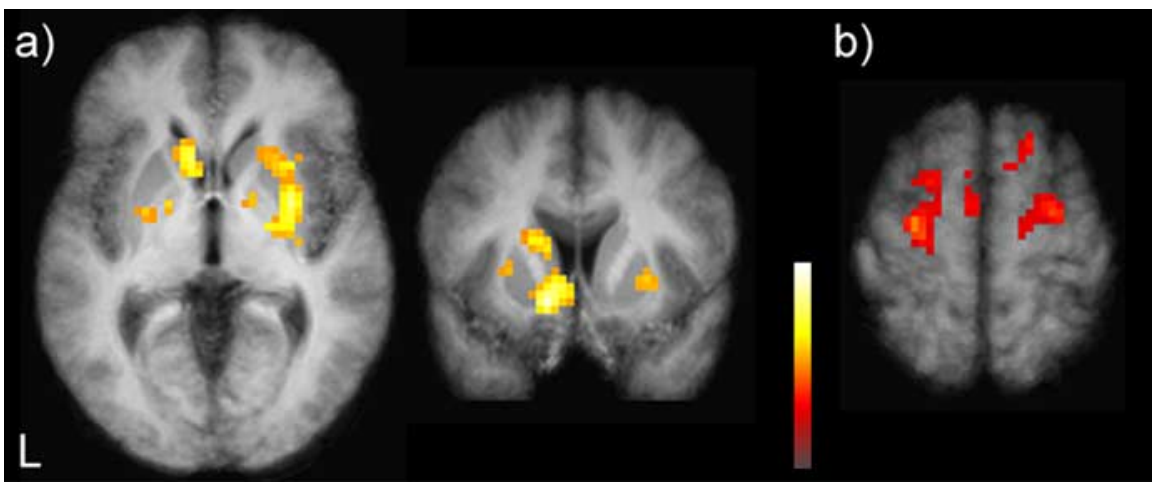

Figure 3. Basal ganglia and frontal cortical areas showing RAC displacement (i.e., DA release) during learning. For illustration purposes, only results projecting on BG $(\boldsymbol{a})$ and frontal cortical motor areas $(\boldsymbol{b})$ are shown (at $p<0.001$ and $p<0.05$, uncorrected, respectively). Results are displayed on axial (left and right panels) and coronal (middle panel) views of the mean PETcoregistered MRI image. The pseudocolor scale represents $T$ values (range, $0-8$ ). Voxel peak coordinates are given in supplemental Tables 1 and 2, available at www.jneurosci.org as supplemental material. The figure was created using MASCOI, freely available at http://homepages.uni-tuebingen.de/matthias.reimold/mascoi/. L, Left. test. Given our a priori hypothesis in BG and caudal frontal cortex, results in these regions were considered as significant at $P_{\mathrm{SVC}}$ $<0.05$ (after indicates FWE correction for multiple comparisons in a 5 -mm-radius spherical volume centered on the peak). At this threshold, significant decreases in RAC BP during LEARNING were observed in striatal and extrastriatal regions (Fig. 3) at both subcortical (supplemental Table 1, available at www.jneurosci. org as supplemental material) and cortical levels (supplemental Table 2, available at www.jneurosci.org as supplemental material).

We also tentatively report decreased RAC BP in the left medial temporal cortex $(x / y / z=-27 /-6 /-24 \mathrm{~mm} ; Z$ score $=4.19)$ and upper midbrain $(x / y / z=3 /-30 /-15 \mathrm{~mm} ; Z$ score $=4.21)$, but those results were not discussed because they were observed in areas that were not specified a priori ( $p>0.05$, after correction for multiple comparisons).

\section{Correlation between behavioral and PET data}

Next, a voxel-by-voxel correlation analysis was performed between DIFF_SLOPE and images representing the magnitude of the difference in RAC BP between conditions (BASELINE minus LEARNING). By applying the same procedure as that described above, we found a significant negative association in the sensorimotor part of the GPi $(x / y / z=-18 /-3 /-6 \mathrm{~mm} ; Z$ score $=3.54$; $\left.P_{\text {SVC }}=0.004\right)($ Fig. $4 a)$ in the hemisphere contralateral to the moving fingers, meaning that faster learning was related to lower DA release in this region. No positive correlation was observed in any BG subdivisions (including ventral striatum) even at very permissive statistical thresholds ( $p<0.05$, uncorrected). In motor-related cortical regions, we found a significant positive association with DIFF_SLOPE in the medial frontal gyrus [medial premotor cortex - pre-supplementary motor area (preSMA); $x / y / z=0 / 6 / 60 \mathrm{~mm} ; Z$ score $\left.=3.13, P_{\mathrm{SVC}}=0.016\right]$ (Picard and Strick, 1996). At $p<0.001$, uncorrected, a correlation was observed in a few other cortical areas but those results are not discussed because they were observed in areas that were not specified a priori.

Post hoc VOI analyses

PET data were analyzed post hoc using a VOI approach (supplemental Tables 3, 4, available at www.jneurosci.org as supplemental material).

Individual percentage changes in RAC BP between BASELINE and LEARNING in specific VOIs were computed as follows (Watabe et al., 2000): Delta_BP $=\left[\left(\mathrm{BP}^{\text {BASELINE }}-\mathrm{BP}^{\text {LEARNING }}\right) /\right.$ $\left.\mathrm{BP}^{\text {BASELINE }}\right] \times 100$.

The group mean Delta_BP in pre-SMA was $7.4 \%$, suggesting that DA was released in this region although this did not reach statistical significance because of the relatively large betweensubjects variability. We observed a positive correlation between DIFF_SLOPE and Delta_BP in the pre-SMA VOI $(r=0.54 ; p=$ $0.04)$, whereas a negative correlation was found in the left GPi VOI (Pearson's $r=-0.66 ; p=0.007$ ) (Fig. $4 b$ ). This post hoc correlation analysis remains significant after discarding data from the left-handed subject both for the pre-SMA VOI $(r=$ $0.55, p=0.04$ ) and the left GPi VOI (Pearson's $r=-0.57, p=$ $0.03)$. A VOI analysis focused on the SMA proper, a motorrelated area caudal to pre-SMA that also receives dopaminergic inputs, did not yield significant results (Pearson's $r=-0.34 ; p=$ 0.21 ). In addition, no significant result was observed for a VOI centered on the left posterior putamen (Pearson's $r=0.02 ; p=$ $0.94)$. This confirms the results of statistical parametric mapping analyses.

The group mean absolute activity in cerebellum $( \pm \mathrm{SD})$ after normalization to the injected activity was $12.4 \pm 2.7(\mathrm{nCi} / \mathrm{ml} /$ $\mathrm{mCi}$ injected) during the BASELINE condition and $12.3 \pm 2.3$ ( $\mathrm{nCi} / \mathrm{ml} / \mathrm{mCi}$ injected) during the LEARNING condition. This difference was not significant (paired $t$ test, $p=0.49$ ). Hence, learning has no significant effect on the overall cerebellar cortex activity, which was used as a reference for computing RAC BP values.

We found no significant negative correlation between Delta _BP and RAC BP values across subjects either during the BASELINE or the LEARNING conditions. In another set of correlation analyses, we found no significant positive relationship between DIFF_SLOPE and BP values during LEARNING or BASELINE conditions. In summary, smaller Delta_BP values and slower learning were not associated with higher BP values (i.e., ceiling effect) in the brain areas examined.

\section{Discussion}

We used RAC PET to measure changes in DA levels in striatal and extrastriatal areas involved in motor control while 15 healthy individuals performed an implicit motor learning task compared with a baseline task. When LEARNING was compared with BASELINE, a significant displacement of RAC (i.e., decrease in 

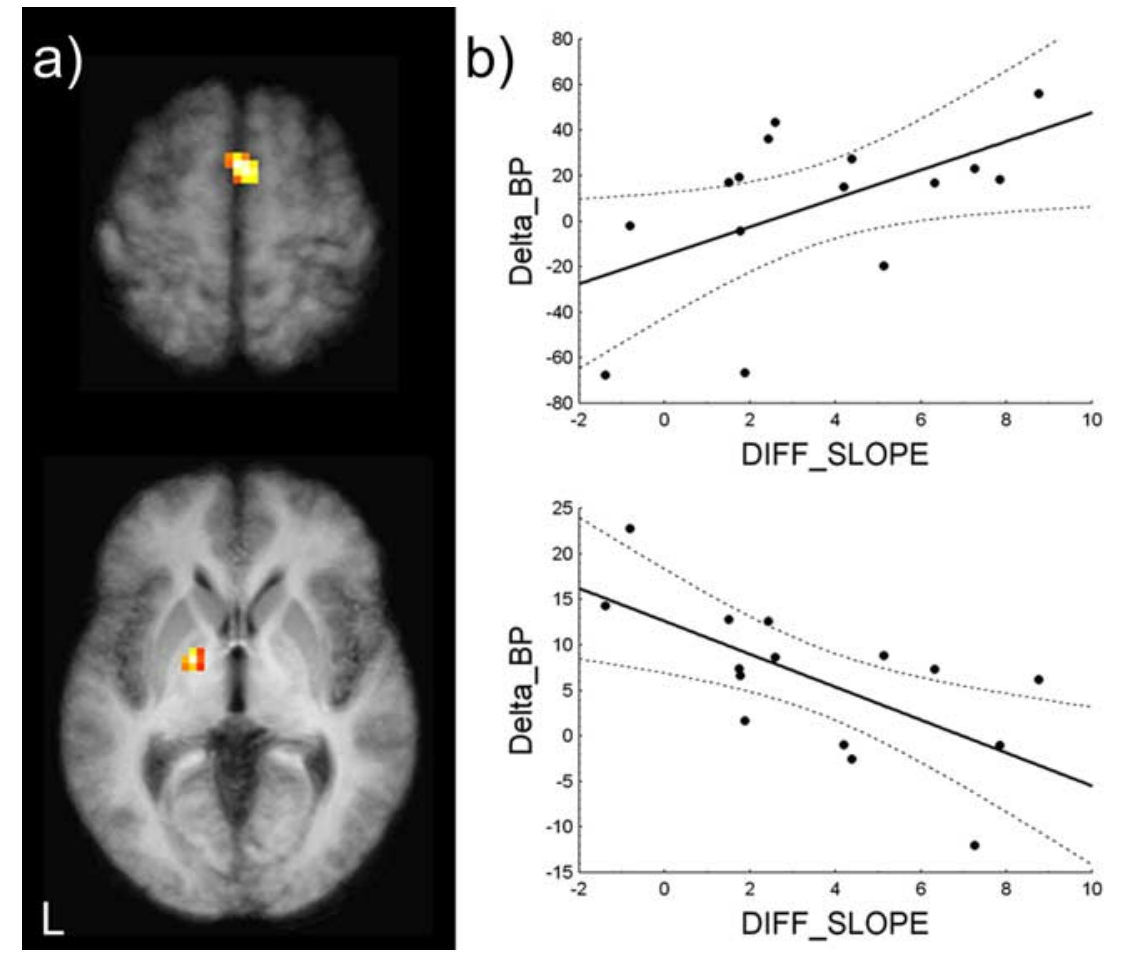

Figure 4. Results of the correlation analyses between behavioral and imaging data. $\boldsymbol{a}$, Voxel-based analysis. Areas showing a positive and a negative correlation between RAC displacement (i.e., DA release) and DIFF_SLOPE are shown in the top and bottom row, respectively. For illustration purposes, only results projecting on $\mathrm{BG}$ and cortical motor areas are shown (at $p<0.05$, uncorrected). The figure was created using MASCOI, freely available at http://homepages.uni-tuebingen.de/matthias.reimold/ mascoi/. L, Left. $\boldsymbol{b}$, Post hoc VOI analysis. The relationship between percentage RAC BP decreases during LEARNING compared with BASELINE (Delta_BP) and DIFF_SLOPE in VOIs centered on medial premotor cortex (pre-SMA, top row) and left GPi (bottom row). High, positive values on the ordinate and abscissa axes indicate a higher RAC displacement (i.e., DA release) in the corresponding brain area and faster learning, respectively. The regressions are shown with $95 \%$ confidence bands.

RAC BP) was observed not only in the striatum as predicted by previous reports (Goerendt et al., 2003) but also in extrastriatal motor circuits, bilaterally, suggesting DA release in these regions (Fig. 3). To increase the specificity of our results for finger movements, the BASELINE condition was designed as an oculomotor sequence task. However, perfect behavioral matching during the two condition-specific PET measurements is obviously difficult to achieve as a result of the difference in time required to perform these measurements (Fig. 2). The estimated BP values were not vulnerable to cerebral blood flow $(\mathrm{CBF})$ variations, which inevitably occurred between subjects (Peigneux et al., 2000) and experimental conditions. By using a bolus-plus-infusion paradigm for RAC administration, $\mathrm{CBF}$, among other factors, controls the speed at which the RAC signal reaches its equilibrium level but not the final activity level (Carson, 2000). Finally, as in previous RAC PET studies, differences in condition-specific BP values are related to an integral of extracellular DA over time so that rapid DA releases are indistinguishable from small prolonged releases (Endres et al., 1997; Endres and Carson, 1998).

RAC displacement in striatum agrees well with results from previous experiments showing bilateral DA release in this region during execution of sequential finger movements (Goerendt et al., 2003). Percentage changes in RAC BP between experimental conditions in these areas are in the same range as those previously reported. The nonuniform pattern of DA release within BG is consistent with previous human PET studies (Strafella et al., 2001, 2003; Goerendt et al., 2003; Zald et al., 2004) as well as in vivo microdialysis and voltammetry animal experiments (Iwano et al., 1997; Cragg et al., 2002). In addition to action potential occurrence (Westerink et al., 1987), the amount of endogenous DA released regionally is known to reflect the influence of several other factors including presynaptic neuronal inputs (Cheramy et al., 1986) and activation of glutamate receptors (Zhang and Sulzer, 2003).

Here, no correlation could be demonstrated between RAC displacement in the striatum and motor learning speed during PET as indexed by DIFF_SLOPE. This discrepancy, with a large number of previous studies supporting a role for DA in motor learning, may arise from differences in the task paradigm and outcome measure of learning. Striatal DA activity has been previously implicated in habit formation and long-term storage and retrieval of well learned movements, especially when learning is associated with rewarding stimuli (Koepp et al., 1998; Matsumoto et al., 1999; Reynolds et al., 2001). Here, no reward (i.e., monetary incentive) was given to the participants as a function of their performance level. Furthermore, DIFF_SLOPE was related to high-order aspects of skill acquisition (see Introduction), and motor learning was examined at its early stage (Karni et al., 1998) as corroborated by the large improvement in behavioral performance during PET compared with that observed the day after (Table 1). Thus, we cannot exclude the possibility that RAC displacement we have observed in different striatal territories is specifically linked to motor learning but that this relationship is not well captured by our experimental design.

An important contribution of this work is the observed RAC displacement (i.e., DA release) in extrastriatal regions bilaterally despite lower RAC BP and consequently lower signal-to-noise ratio (SNR) in these areas compared with striatum. Anatomical studies in primates showed that the involved areas, GPi and premotor cortex, receive strong DA inputs from midbrain DA neurons (Williams and Goldman-Rakic, 1998; Jan et al., 2000). In humans, successful extrastriatal RAC BP measurements have been previously reported including in rostral frontal areas (Piccini et al., 2003), which are less densely innervated by DA than the more caudal premotor regions (Gaspar et al., 1989). Spatial image smoothing in voxel-based analyses, as in the present study, may increase SNR and gives additional power to detect subtle changes in regional RAC BP (Piccini et al., 2003). Furthermore, there is also some evidence suggesting that the power to measure RAC changes using a single-scan approach, as in the present study, is higher than using the dual-scan approach (Marenco et al., 2004).

As opposed to previous studies, our finding in extrastriatal regions is further validated by showing a significant correlation with behavioral data (Fig. 4). Whereas RAC displacement was observed in GPi, bilaterally, a relationship with DIFF_SLOPE was only observed with the sensorimotor region of the GPi contralateral to the moving hand (by convention here, the left GPi), in agreement with known organization of the motor system. Avail- 
able evidence suggests that the GPi is strongly involved in motor control (Boecker et al., 1998) including motor skill learning (Thompson et al., 1987; Carbon et al., 2003). Although innervation of GPi (or its equivalent in rodents, the nucleus entopeduncularis) by midbrain DA neurons is well established (Jan et al., 2000), the functional role of this anatomical pathway has received little attention to date (Whone et al., 2003).

Here, RAC displacement in left GPi was inversely correlated to DIFF_SLOPE. In other words, in our group of healthy individuals, faster learning was associated with lower DA release in left GPi. After our working hypothesis that DA release in interconnected BG and cortical areas shows an opposite relationship with behavior, we looked for a region in the caudal frontal cortex in which DA levels increased as a function of better performance levels. We found a significant result in the pre-SMA (Picard and Strick, 1996), a cortical medial premotor area that has strong anatomical (Sakai et al., 2000) and functional (Grafton et al., 1995) connections with the GPi. This result supports current theories suggesting that the medial premotor cortex is part of an implicit sequence learning system (Keele et al., 2003). After the proposal that DA may modulate functional cerebral connectivity in a task-dependent manner (Williams et al., 2002), a high, sustained, background dopaminergic tone in pre-SMA in subjects showing faster learning of the probabilistic SRTT may have more efficiently reshaped its functional interactions with other brain areas engaged in the task such as those processing competing action sets during rule-based stimulus-driven motor actions (Bunge, 2004; Rushworth et al., 2004).

The functional significance of the inverse relationship between task-related DA release in interconnected BG and cortical areas with behavior calls for further studies. A general hypothesis about DA function suggests that it may control among competing inputs by locally enhancing the SNR, which may ultimately enable synaptic plasticity in the form of long-term potentiation (Reynolds et al., 2001; O'Donnell, 2003). By reference to an emerging theory supporting competitive mechanisms between cortical and subcortical systems for behavioral control (Fujii and Graybiel, 2005), the present findings might suggest that higher DA levels in pre-SMA may favor cortical as opposed to subcortical learning processes in a task-dependent manner. This interpretation would be consistent with previous studies demonstrating a prominent role of pre-SMA in the early stages of motor skill learning (Grafton et al., 1992, 1994).

The observed interaction between BG and cortical DA systems as a function of task performance may have important implications regarding our understanding of the mechanisms by which behavior is modulated by DA. Although alternative explanations are theoretically possible, these results agree with basic research, which has provided strong support for the concept that DA signaling in the BG is under the control of the frontal cortex. It has been proposed that frontal areas exert a brake on DA signaling in subcortical regions that, in functional terms, are linked to these cortical areas (Carlsson et al., 2000; Sesack et al., 2003). This effect may be modulated by cortical DA. In vivo experiments in rodents and monkeys have demonstrated that pharmacologically or behaviorally induced increases in frontal DA are associated with decreased BG DA release (Kolachana et al., 1995; Iwano et al., 1997). However, an overall, simple reciprocal or inverse relationship in DA release between subcortical and cortical regions has not yet been reported (Wilkinson et al., 1998). This might be caused by differences in the kinetics of DA release between BG and cortical areas (Garris and Wightman, 1994). Nevertheless, we speculate here that the activation of mesocortical neurons pro- jecting to medial premotor cortex may have increased DA locally as a function of improved performance levels, which in turn resulted in an inhibitory influence on DA release in functionally linked subcortical areas such as sensorimotor GPi. The underlying mechanisms are undoubtedly complex and incompletely understood (Carr and Sesack, 2000; Jackson et al., 2001). This interaction may also apply to other anatomically and functionally interconnected BG and frontal cortical areas as a function of different task paradigms.

\section{References}

Boecker H, Dagher A, Ceballos-Baumann AO, Passingham RE, Samuel M, Friston KJ, Poline JB, Dettmers C, Conrad B, Brooks DJ (1998) Role of the human rostral supplementary motor area and the basa ganglia in motor sequence control: investigations with $\mathrm{H}_{2}{ }^{15} \mathrm{O}$ PET. J Neurophysiol 79:1070-1080.

Bunge SA (2004) How we use rules to select actions: a review of evidence from cognitive neuroscience. Cogn Affect Behav Neurosci 4:564-579.

Carbon M, Ghilardi MF, Feigin A, Fukuda M, Silvestri G, Mentis MJ, Ghez C, Moeller JR, Eidelberg D (2003) Learning networks in health and Parkinson's disease: reproducibility and treatment effects. Hum Brain Mapp 19:197-211.

Carlsson A, Waters N, Waters S, Carlsson ML (2000) Network interactions in schizophrenia - therapeutic implications. Brain Res Brain Res Rev 31:342-349.

Carr DB, Sesack SR (2000) Projections from the rat prefrontal cortex to the ventral tegmental area: target specificity in the synaptic associations with mesoaccumbens and mesocortical neurons. J Neurosci 20:3864-3873.

Carson RE (2000) PET physiological measurements using constant infusion. Nucl Med Biol 27:657-660.

Cheramy A, Romo R, Godeheu G, Baruch P, Glowinski J (1986) In vivo presynaptic control of dopamine release in the cat caudate nucleus - II Facilitatory or inhibitory influence of L-Glutamate. Neuroscience 19:1081-1090.

Cragg SJ, Hille CJ, Greenfield SA (2002) Functional domains in dorsal striatum of the nonhuman primate are defined by the dynamic behavior of dopamine. J Neurosci 22:5705-5712.

Duvauchelle CL, Levitin M, MacConell LA, Lee LK, Ettenberg A (1992) Opposite effects of prefrontal cortex and nucleus accumbens infusions of flupenthixol on stimulant-induced locomotion and brain stimulation reward. Brain Res 576:104-110.

Endres CJ, Carson RE (1998) Assessment of dynamic neurotransmitter changes with bolus or infusion delivery of neuroreceptor ligands. J Cereb Blood Flow Metab 18: 1196-1210.

Endres CJ, Kolachana BS, Saunders RC, Su T, Weinberger D, Breier A, Eckelman WC, Carson RE (1997) Kinetic modeling of [11C]raclopride: combined PET-microdialysis studies. J Cereb Blood Flow Metab 17:932-942.

Fujii N, Graybiel AM (2005) Time-varying covariance of neural activities recorded in striatum and frontal cortex as monkeys perform sequentialsaccade tasks. Proc Natl Acad Sci USA 102:9032-9037.

Garris PA, Wightman RM (1994) Different kinetics govern dopaminergic transmission in the amygdala, prefrontal cortex, and striatum: an in vivo voltammetric study. J Neurosci 14:442-450.

Gaspar P, Berger B, Febvret A, Vigny A, Henry JP (1989) Catecholamine innervation of the human cerebral cortex as revealed by comparative immunohistochemistry of tyrosine hydroxylase and dopamine-betahydroxylase. J Comp Neurol 279:249-271.

Goerendt IK, Messa C, Lawrence AD, Grasby PM, Piccini P, Brooks DJ (2003) Dopamine release during sequential finger movements in health and Parkinson's disease: a PET study. Brain 126:312-325.

Grafton ST, Mazziotta JC, Presty S, Friston KJ, Frackowiak RS, Phelps ME (1992) Functional anatomy of human procedural learning determined with regional cerebral blood flow and PET. J Neurosci 12:2542-2548.

Grafton ST, Woods RP, Tyszka JM (1994) Functional imaging of procedural motor learning: relating cerebral blood flow with individual subject performance. Hum Brain Mapp 1:221-234.

Grafton ST, Waters C, Sutton J, Lew MF, Couldwell W (1995) Pallidotomy increases activity of motor association cortex in Parkinson's disease: a positron emission tomographic study. Ann Neurol 37:776-783.

Iwano H, Yamamuro Y, Hori K, Yamauchi T, Nomura M (1997) Effects of 
discrimination learning on the rat striatal dopaminergic activity: a microdialysis study. Neuroreport 8:1095-1098.

Jackson ME, Frost AS, Moghaddam B (2001) Stimulation of prefrontal cortex at physiologically relevant frequencies inhibits dopamine release in the nucleus accumbens. J Neurochem 78:920-923.

Jan C, Francois C, Tande D, Yelnik J, Tremblay L, Agid Y, Hirsch E (2000) Dopaminergic innervation of the pallidum in the normal state, in MPTPtreated monkeys and in parkinsonian patients. Eur J Neurosci 12:4525-4535.

Jiménez L, Mendez C, Cleeremans A (1996) Comparing direct and indirect measures of sequence learning. J Exp Psychol Learn Mem Cogn 22:948-969.

Joel D, Weiner I (2000) The connections of the dopaminergic system with the striatum in rats and primates: an analysis with respect to the functional and compartmental organization of the striatum. Neuroscience 96:451-474.

Karni A, Meyer G, Rey-Hipolito C, Jezzard P, Adams MM, Turner R, Ungerleider LG (1998) The acquisition of skilled motor performance: fast and slow experience-driven changes in primary motor cortex. Proc Natl Acad Sci USA 95:861-868.

Keele SW, Ivry R, Mayr U, Hazeltine E, Heuer H (2003) The cognitive and neural architecture of sequence representation. Psychol Rev 110:316-339.

Koepp MJ, Gunn RN, Lawrence AD, Cunningham DA, Jones T, Brooks DJ, Bench CJ, Grasby PM (1998) Evidence for striatal dopamine release during a video game. Nature 393:266-268.

Kolachana BS, Saunders RC, Weinberger DR (1995) Augmentation of prefrontal cortical monoaminergic activity inhibits dopamine release in the caudate nucleus: an in vivo neurochemical assessment in the rhesus monkey. Neuroscience 69:859-868.

Laruelle M (2000) Imaging synaptic neurotransmission with in vivo binding competition techniques: a critical review. J Cereb Blood Flow Metab 20:423-451.

Lungu OV, Wachter T, Liu T, Willingham DT, Ashe J (2004) Probability detection mechanisms and motor learning. Exp Brain Res 159:135-150.

Marenco S, Carson RE, Berman KF, Herscovitch P, Weinberger DR (2004) Nicotine-induced dopamine release in primates measured with [11C]raclopride PET. Neuropsychopharmacology 29:259-268.

Matsumoto N, Hanakawa T, Maki S, Graybiel AM, Kimura M (1999) Role of nigrostriatal dopamine system in learning to perform sequential motor tasks in a predictive manner. J Neurophysiol 82:978-998.

Mawlawi O, Martinez D, Slifstein M, Broft A, Chatterjee R, Hwang DR, Huang Y, Simpson N, Ngo K, Van Heertum R, Laruelle M (2001) Imaging human mesolimbic dopamine transmission with positron emission tomography: I. Accuracy and precision of $\mathrm{D}_{2}$ receptor parameter measurements in ventral striatum. J Cereb Blood Flow Metab 21:1034-1057.

Meyer JH, Gunn RN, Myers R, Grasby PM (1999) Assessment of spatial normalization of PET ligand images using ligand-specific templates. Neuroimage 9:545-553.

Nissen MJ, Bullemer P (1987) Attentional requirements of learning: evidence from performances measures. Cogn Psychol 19:1-32.

O’Donnell P (2003) Dopamine gating of forebrain neural ensembles. Eur J Neurosci 17:429-435.

Oldfield RC (1970) The assessment and analysis of handedness: the Edinburgh inventory. Neuropsychologia 9:97-113.

Peigneux P, Maquet P, Meulemans T, Destrebecqz A, Laureys S, Degueldre C, Delfiore G, Aerts J, Luxen A, Franck G, Van der Linden M, Cleeremans A (2000) Striatum forever, despite sequence learning variability: a random effect analysis. Hum Brain Mapp 10:179-194.

Picard N, Strick PL (1996) Motor areas of the medial wall: a review of their location and functional activation. Cereb Cortex 6:342-353.
Piccini P, Pavese N, Brooks DJ (2003) Endogenous dopamine release after pharmacological challenges in Parkinson's disease. Ann Neurol 53:647-653.

Pycock CJ, Carter CJ, Kerwin RW (1980) Effect of 6-hydroxydopamine lesions of the medial prefrontal cortex on neurotransmitter systems in subcortical sites in the rat. J Neurochem 34:91-99.

Reynolds JN, Hyland BI, Wickens JR (2001) A cellular mechanism of reward-related learning. Nature 413:67-70.

Roberts AC, De Salvia MA, Wilkinson LS, Collins P, Muir JL, Everitt BJ, Robbins TW (1994) 6-Hydroxydopamine lesions of the prefrontal cortex in monkeys enhance performance on an analog of the Wisconsin Card Sort Test: possible interactions with subcortical dopamine. J Neurosci 14:2531-2544.

Rushworth MF, Walton ME, Kennerley SW, Bannerman DM (2004) Action sets and decisions in the medial frontal cortex. Trends Cogn Sci 8:410-417.

Sakai ST, Stepniewska I, Qi HX, Kaas JH (2000) Pallidal and cerebellar afferents to pre-supplementary motor area thalamocortical neurons in the owl monkey: a multiple labeling study. J Comp Neurol 417:164-180.

Sesack SR, Carr DB, Omelchenko N, Pinto A (2003) Anatomical substrates for glutamate-dopamine interactions: evidence for specificity of connections and extrasynaptic actions. Ann NY Acad Sci 1003:36-52.

Strafella A, Paus T, Barrett J, Dagher A (2001) Repetitive transcranial stimulation of the human prefrontal cotex induces dopamine release in the caudate nucleus. J Neurosci 21: RC157.

Strafella AP, Paus T, Fraraccio M, Dagher A (2003) Striatal dopamine release induced by repetitive transcranial magnetic stimulation of the human motor cortex. Brain 126:2609-2615.

Thieben MJ, Duggins AJ, Good CD, Gomes L, Mahant N, Richards F, McCusker E, Frackowiak RS (2002) The distribution of structural neuropathology in pre-clinical Huntington's disease. Brain 125:1815-1828.

Thompson R, Huestis PW, Yu J (1987) Motor learning: nonspecific subcortical mechanisms in rats. Arch Phys Med Rehabil 68:419-422.

Watabe H, Endres CJ, Breier A, Schmall B, Eckelman WC, Carson RE (2000) Measurement of dopamine release with continuous infusion of [11C]raclopride: optimization and signal-to-noise considerations. J Nucl Med 41:522-530.

Westerink BH, Tuntler J, Damsma G, Rollema H, de Vries JB (1987) The use of tetrodotoxin for the characterization of drug-enhanced dopamine release in conscious rats studied by brain dialysis. Naunyn Schmiedebergs Arch Pharmacol 336:502-507.

Whone AL, Moore RY, Piccini PP, Brooks DJ (2003) Plasticity of the nigropallidal pathway in Parkinson's disease. Ann Neurol 53:206-213.

Wilkinson LS, Humby T, Killcross AS, Torres EM, Everitt BJ, Robbins TW (1998) Dissociations in dopamine release in medial prefrontal cortex and ventral striatum during the acquisition and extinction of classical aversive conditioning in the rat. Eur J Neurosci 10:1019-1026.

Williams D, Tijssen M, Van Bruggen G, Bosch A, Insola A, Di L, V, Mazzone P, Oliviero A, Quartarone A, Speelman H, Brown P (2002) Dopaminedependent changes in the functional connectivity between basal ganglia and cerebral cortex in humans. Brain 125:1558-1569.

Williams SM, Goldman-Rakic PS (1998) Widespread origin of the primate mesofrontal dopamine system. Cereb Cortex 8:321-345.

Zald DH, Boileau I, El Dearedy W, Gunn R, McGlone F, Dichter GS, Dagher A (2004) Dopamine transmission in the human striatum during monetary reward tasks. J Neurosci 24:4105-4112.

Zhang H, Sulzer D (2003) Glutamate spillover in the striatum depresses dopaminergic transmission by activating group I metabotropic glutamate receptors. J Neurosci 23:10585-10592. 